\title{
Laparoscopic Surgery vs. Open Surgery for Older Patients with Rectal Cancer: A Comparative Study of Propensity Score
}

Qiang Li (D liqiang2169@163.com )

Capital Medical University Affiliated Beijing Shijitan Hospital https://orcid.org/0000-0001-6901-1291 Hong Gao

Capital Medical University Affiliated Beijing Shijitan Hospital

Jin-Zhong Wang

Capital Medical University Affiliated Beijing Shijitan Hospital

Yu-Bing Zhu

Capital Medical University Affiliated Beijing Shijitan Hospital

\section{Research}

Keywords: laparoscopic surgery, rectal cancer, older patients

Posted Date: July 26th, 2021

DOI: https://doi.org/10.21203/rs.3.rs-725883/v1

License: (c) (i) This work is licensed under a Creative Commons Attribution 4.0 International License. Read Full License 


\section{Abstract}

Background The purpose of this study was to compare the efficacy of laparoscopic surgery to open rectal cancer surgery in patients aged 70 years and older.

Methods Consecutive patients ( $\geq 70$ years old) who underwent rectal cancer surgery were retrospectively selected. Based on the covariates (age, gender, body mass index (BMI), ASA, TNM staging), the propensity score was calculated for paired comparison. The Tumor Distance from the Anal Verge (TDAV), history of abdominal surgery, combined diseases, time of operation, amount of blood loss, anastomosis method, postoperative hospital stay, time to first exhaust, time to first oral diet, postoperative complications, postoperative 30-day mortality and postoperative pathological staging of two groups were collected.

Results Shorter operating time, higher intraoperative blood loss, slower intestinal function, longer time to first exhaust, longer postoperative hospital stay, higher incidence of postoperative complications (no statistical difference), and shorter duration of analgesics were observed in the OS group (all $P<0.05)$. The sphincter preserving rate was basically the same in the two groups. For low rectal cancer with TDAV $<5$ $\mathrm{cm}$, more patients tended to choose LS ( $P=0.051)$; for middle rectal cancer with TDAV of 5-10 cm, more patients tended to choose OS $(P=0.032)$. There was no significant difference in postoperative survival rate between the two groups.

Conclusions In older patients, laparoscopic rectal cancer surgery led to less trauma, and a faster recovery compared to open surgery, with significant short-term advantages and no significant long-term advantages. For low rectal cancer, the advantage of LS was more prominent.

\section{Introduction}

Colorectal cancer is the third most common tumor in the world. According to the World Health Organization (WHO), 1.4 million people worldwide were diagnosed with colorectal cancer in 2012, onethird of which were found in the rectum [1]. In addition, studies have suggested that the incidence of colorectal cancer increases with patient age ${ }^{[2]}$. For example, in China, the incidence of colorectal cancer among people over 75 years is about 78,200 per year, accounting for $18.08 \%$ of the global incidence ${ }^{[3]}$.

Laparoscopic surgery had been widely applied for the treatment of colorectal cancer. Compared to traditional open surgery, it offers similar therapeutic efficiency with less trauma and pain and faster recovery ${ }^{[4-9]}$.

Chronic diseases, such as cardiovascular disease and diabetes, are commonly observed in older patients. These diseases impair body function and significantly increase the risk of surgery. Age $>70$ years old is also considered as an independent predictor of increased risk of postoperative complications, with higher postoperative mortality and longer hospital stay ${ }^{[10 / 11 / 12]}$. The purpose of this study was to compare the status and efficacy of laparoscopic and open rectal cancer surgery in patients aged 70 years and older. 


\section{Materials And Methods \\ Patients}

A retrospective study was conducted on consecutive rectal cancer patients who underwent surgery in Beijing Shijitan Hospital from January 2015 to September 2019. The inclusion criteria were the following: patients older than 70 years who underwent colonoscopy before the operation and were pathologically diagnosed with rectal cancer. CT and MRI performed preoperative pathological staging (the 8th edition of UICC TNM staging system), and surgical indications were confirmed. The heart, lung, liver, and kidney functions were evaluated before surgery, and no contraindications were confirmed. The patients underwent radical surgery for rectal cancer. Cases that required emergency surgery, palliative surgery, and preoperative neoadjuvant therapy were excluded.

The patients were divided into two groups: a study group, which included patients who received laparoscopic surgery (LS), and a control group that included patients who underwent open surgery (OS). Patients who started laparoscopic surgery and switched to open surgery during the operation were excluded.

The study protocol has been approved by The Ethics Committee for Scientific Research of Capital Medical University Affiliated Beijing Shijitan Hospital and the research meets requirements for protection of human subjects.

\section{Clinical Data Collection}

Preoperative observation indicators included age, gender, body mass index (BMI), American Society of Anesthesiologists (ASA), Tumor Distance from the Anal Verge (TDAV), history of abdominal surgery, and combined disease. The combined disease was assessed using the Charlson Comorbidity Index (CCl) ${ }^{[13]}$. Intraoperative observation indicators included operation time, blood loss, and anastomosis method. Postoperative observation indicators included postoperative hospital stay, time to first exhaust, time to first oral diet, postoperative complications, postoperative 30-day mortality, and postoperative pathological staging (the 8th edition of UICC TNM staging system).

\section{Survival Outcomes Collection}

The patients were followed up by outpatient visits, telephone calls, or home visits. Patients who received outpatient follow-up were revisited once every 3 months for 2 years after surgery. After 2 years of surgery, they were reviewed every 6 months. The outpatient review included consultation, physical examination, tumor marker examination, and imaging assessment. Colonoscopy was performed once every 6 months. The survival status of some patients who could not receive outpatient follow-up was collected by 
telephone or during home visits. The primary endpoint was death from recurrence and metastasis of rectal cancer, and the secondary endpoint was September 30, 2019.

\section{Statistical analysis}

SPSS 21.0 software was used for statistical analysis (IBM-SPSS, Chicago, USA.). According to the covariates (age, gender, BMI, ASA, TNM staging), the multivariate Logistic retrospective model was used to calculate each patient's propensity score. Each patient in the OS group was matched with a case with the closest score in the LS group to form a pair. The basic data and surgical results of the two groups of patients were compared using a paired t-test. Wilcoxon signed ranks test was used for continuous variables. McNemar's test was used for proportional data. A paired model with more than two levels based on propensity scores was used for categorical variables. Kaplan-Meier analysis was used for survival analysis. $P<0.05$ was considered statistically significant.

\section{Results}

\section{Patient characteristics}

Among a total of 671 rectal cancer surgeries that were performed in our hospital between January 2015 and September 2019, 198 cases were over 70 years old. Among them, 63 cases were of open surgery and 135 cases of laparoscopic surgery. After excluding patients who underwent emergency surgery, palliative surgery, and preoperative neoadjuvant therapy, 51 patients who underwent open surgery were enrolled. In addition, 121 cases of laparoscopic surgery were finally included after excluding 4 cases that converted to laparotomy during operation. The pairing was performed according to the covariate; each open surgery case was paired with the most similar laparoscopic surgery case, with a total of 51 pairs.

The demographics of the two groups of patients are shown in Table 1. The age of patients in the two groups was $76.6 \pm 4.6$ years old and $77.1 \pm 5.3$ years old, respectively. The results of age, gender, BMI, ASA, and TNM staging were similar, and there was no statistical difference between the two groups. The $\mathrm{CCl}$ of patients in the two groups was also similar $(P=0.920)$. The proportion of comorbidities in both groups was higher. In the LS group, $25.5 \%(n=13)$ patients had no combined disease, while $23.5 \%(n=$ 12) patients had no combined disease in the OS group $(\mathrm{CCl}=0)$. The ASA score of $51.0 \%(n=26)$ patients in LS group was 3 or 4 points, with $49.0 \%(n=25)$ in the OS group. 
Table 1

Patient demographics of LS and OS group

\begin{tabular}{|c|c|c|c|}
\hline & LS group $(n=51)$ & OS group $(n=51)$ & $P$-value \\
\hline Age (years) & $77.5 \pm 4.5$ & $76.5 \pm 5.0$ & 0.309 \\
\hline Gender. F/M & $19 / 32$ & $19 / 32$ & 1.000 \\
\hline BMI & $23.7 \pm 0.9$ & $23.8 \pm 1.3$ & 0.814 \\
\hline ASA & & & 0.453 \\
\hline 2 & $25(49.0 \%)$ & $26(51.0 \%)$ & \\
\hline 3 & $18(35.3 \%)$ & $21(41.2 \%)$ & \\
\hline 4 & $8(15.7 \%)$ & $4(7.8 \%)$ & \\
\hline TMN & & & 0.217 \\
\hline ૧ & $4(7.8 \%)$ & $6(11.8 \%)$ & \\
\hline ૫ & $22(43.1 \%)$ & $24(47.1 \%)$ & \\
\hline 口 & $21(41.2 \%)$ & $23(45.1 \%)$ & \\
\hline$\square$ & $4(7.8 \%)$ & $0(0 \%)$ & \\
\hline $\mathrm{CCl}$ & & & 0.920 \\
\hline 0 & $13(25.5 \%)$ & $12(23.5 \%)$ & \\
\hline 1 & $16(31.4 \%)$ & $1(2.0 \%)$ & \\
\hline 2 & $18(35.3 \%)$ & $17(33.3 \%)$ & \\
\hline 3 & $3(5.9 \%)$ & $5(9.8 \%)$ & \\
\hline 4 & $1(2.0 \%)$ & $2(3.9 \%)$ & \\
\hline
\end{tabular}

\section{Surgical Outcomes}

The OS group and the LS group had similar previous surgery history. The operation time of the OS group was 20 minutes faster than the LS group, and the difference between the two groups was statistically significant $(P=0.048)$. More estimated blood loss was observed in the OS group than in the LS group $(140 \pm 48 \mathrm{ml}$ vs. $119 \pm 51 \mathrm{ml} ; \mathrm{P}=0.027)$. Patients in the LS group showed faster recovery of the postoperative intestinal function and had a shorter time to first exhaust compared to the OS group (64.08 \pm 14.16 hours vs. $82.32 \pm 12.00$ hours; $P<0.001$ ). The LS group's postoperative hospital stay was $10.67 \pm$ 
2.62 days, which was significantly shorter than that of the OS group $(11.86 \pm 2.31$ days $)(P=0.016)$. Moreover, the number of Miles operations in the OS group was more than that in the LS group (10 vs. 8), and the sphincter preserving rate of the OS group was lower, but there was no statistical difference $(\mathrm{P}=$ 0.786) (Table 2).

Table 2

Surgical and oncological outcomes of LS and OS group

\begin{tabular}{|llll|}
\hline & LS group $(\mathbf{n = 5 1 )}$ & OS group $(\mathbf{n}=\mathbf{5 1})$ & P-value \\
\hline Previous surgeries & $22(43.1 \%)$ & $24(47.1 \%)$ & 0.691 \\
\hline Operation time (min) & $193 \pm 28$ & $203 \pm 20$ & 0.048 \\
\hline EBL $(\mathrm{ml})$ & $119 \pm 51$ & $140 \pm 48$ & 0.027 \\
\hline Mode of anastomosis & & & 0.786 \\
\hline Miles & $8(15.7 \%)$ & $10(19.6 \%)$ & \\
\hline Dixon & $40(78.4 \%)$ & $37(72.5 \%)$ & \\
\hline Hartmann & $3(5.9 \%)$ & $4(7.8 \%)$ & \\
\hline Duration of analgesics administered (day) & $1.29 \pm 0.46$ & $1.78 \pm 0.64$ & $\varangle 0.001$ \\
\hline Time to first flatus (h) & $64.08 \pm 14.16$ & $82.32 \pm 12.00$ & $\varangle 0.001$ \\
\hline Time to first oral diet (day) & $3.67 \pm 0.59$ & $4.43 \pm 0.50$ & $\varangle 0.001$ \\
\hline Postoperative stay (day) & $10.67 \pm 2.62$ & $11.86 \pm 2.31$ & 0.016 \\
\hline LS Laparoscopic Surgery; OS Open Surgery; $E B L$ estimated blood loss & \\
\hline
\end{tabular}

\section{Postoperative Complications}

In this study, four major complications occurred in the OS group and LS group, respectively. In the LS group, 2 cases suffered from anastomotic leakage and 2 from intraluminal bleeding; no wound dehiscence was observed. In the OS group, wound dehiscence was observed in 2 cases, 1 case suffered anastomotic leakage, and 1 had intraluminal bleeding. There was no statistical difference in major postoperative complications between the two groups $(P=0.558)$. The incidence of postoperative cardiac failure and pneumonia was higher in both groups. In addition, In the OS group, there were 2 cases of cardiac failure and 4 cases of pneumonia; in the LS group, there were 2 cases of cardiac failure and 2 cases of pneumonia. The incidence of postoperative complications in the OS group was higher than that in the LS group ( $21.6 \%$ vs. $35.3 \%)$, but the difference between the two groups was not statistically significant $(P=0.124)$. There was no death in the two groups within 30 days after the operation (Table 3$)$. 
Table 3

Postoperative complications of LS and OS group

\begin{tabular}{|llll|}
\hline & LS group ( $\mathbf{n}=\mathbf{5 1})$ & OS group ( $\mathbf{n}=\mathbf{5 1})$ & $P$-value \\
\hline Major complications & & & \\
\hline Anastomotic leakage(Dixon) & $2(3.9 \%)$ & $1(2.0 \%)$ & 0.558 \\
\hline Intraluminal bleeding(Dixon) & $2(3.9 \%)$ & $1(2.0 \%)$ & 0.558 \\
\hline Wound dehiscence & $0(0 \%)$ & $2(3.9 \%)$ & 0.153 \\
\hline Minor complications & & & \\
\hline Paralytic ileus & $2(3.9 \%)$ & $4(7.8 \%)$ & 0.400 \\
\hline Wound infection & $1(2.0 \%)$ & $4(7.8 \%)$ & 0.169 \\
\hline Pneumonia & $2(3.9 \%)$ & $4(7.8 \%)$ & 0.400 \\
\hline Acute cardiac failure & $2(3.9 \%)$ & $2(3.9 \%)$ & 1.000 \\
\hline Overall & $11(21.6 \%)$ & $18(35.3 \%)$ & 0.124 \\
\hline LS Laparoscopic Surgery; OS Open Surgery & & \\
\hline
\end{tabular}

\section{Preventative Ostomy Results}

Dixon operation was the main surgical method in both groups (78.4\% vs. $72.5 \%)$. Taking Tumor Distance from the Anal Verge (TDAV) as the dividing standard, we found the following: when TDAV was $<5 \mathrm{~cm}$, Dixon operation in the LS group was higher than the OS group (42.5\% vs. $21.6 \%$ ), but there was no statistical difference between the two groups $(P=0.051)$. When TDAV was $5-10 \mathrm{~cm}$, Dixon operation in the OS group was higher than that in the LS group (51.4\% vs. 27.5\%), and the difference between the two groups was statistically significant $(P=0.032)$. When TDAV $>10 \mathrm{~cm}$, Dixon operation in the LS group and OS group was similar ( $30 \%$ vs. $27 \% ; P=0.773)$.

In Dixon surgery, the proportion of preventative ostomy (PO) in the LS group was higher than that in the OS group (27.5\% vs. $13.5 \%$ ). Most patients in the two groups underwent $\mathrm{PO}$ when TDAV $<5 \mathrm{~cm}$ and the proportion of PO in the LS group was significantly higher than that in the OS group (52.9\% vs. $37.5 \%)$. Still, the difference between the two groups was not statistically significant $(P=0.082)$ (Table 4). 
Table 4

Tumor location about Dixon

\begin{tabular}{|llll|}
\hline & LS group $(\mathbf{n}=\mathbf{4 0 , 7 8 . 4 \%})$ & OS group $(\mathbf{n = 3 7 , 7 2 . 5 \% )}$ & P-value \\
\hline TDAV $\$ 5 \mathrm{~cm}$ & $17(42.5 \%)$ & $8(21.6 \%)$ & 0.051 \\
\hline PO & $9(52.9 \%)$ & $3(37.5 \%)$ & 0.082 \\
\hline TDAV $5-10 \mathrm{~cm}$ & $11(27.5 \%)$ & $19(51.4 \%)$ & 0.032 \\
\hline PO & $2(18.1 \%)$ & $2(10.5 \%)$ & 0.936 \\
\hline TDAV $10 \mathrm{~cm}$ & $12(30 \%)$ & $10(27 \%)$ & 0.773 \\
\hline PO & $0(0 \%)$ & $0(0 \%)$ & 0.000 \\
\hline
\end{tabular}

\section{Follow-up}

The mean follow-up time of patients in the two groups was 35.7 months (7-59 months), with 36.4 months in the OS group and 34.9 months in the LS group. Twenty-two of the 102 patients $(21.6 \%)$ died during the entire follow-up period. The OS group's median survival time was 55 months, and that of the LS group was 53 months. Log-rank test of survival rate between the two groups showed no statistical difference $(\chi 2=0.198, P=0.656)$ (Fig. 1).

\section{Discussion}

With the continuous improvement of sanitary conditions and dietary habits, the world's older population (aged 65 and over) continues to grow at an unprecedented rate, especially in developing countries ${ }^{[14]}$. Yet, with age, the incidence of cancer has also been drastically increasing.

Colorectal cancer is the third most common cause of cancer morbidity in both men and in women. The incidence of colorectal cancer in the population over 75 years is at about 40 to 50 per 100,000 persons compared to about 5 to 20 per 100,000 in persons $60-65$ years ${ }^{[15]}$. Radical surgery is still considered the main treatment for this type of malignancy. However, with the increase of age, the body's reserve capacity gradually decreases, resulting in drastically reduced tolerance to surgery. Devoto et al ${ }^{[16]}$ found that the postoperative mortality and complication rate was significantly increased in patients over 70 years old who undergo colorectal cancer surgery. Moreover, Arenal-Vera and colleagues showed that among patients who were 70 years old or older, the 30 -day mortality rate after colorectal cancer was about $6 \%$, and about $20 \%$ of patients had one or more complications ${ }^{[17]}$. 
Laparoscopic surgery has shown similar long-term efficacy as open surgery in the treatment of colorectal tumors ${ }^{[18]}$. Yet, compared to open surgery, laparoscopic surgery has been associated with less trauma and lower postoperative stress. In addition, lower C-reactive protein and interleukin-6 levels were found in those who underwent laparoscopic surgery ${ }^{[19]}$. However, considering the long operation time and the risk of reduced cardiac output and postoperative atelectasis caused by pneumoperitoneum, the application of laparoscopic surgery in older patients with cancer is still limited ${ }^{[20]}$. Thus, data on laparoscopic rectal cancer surgery in older patients are limited.

In this study, we examined laparoscopic efficacy compared to open rectal cancer surgery in patients aged 70 years and older. Those who did not receive neoadjuvant therapy, emergency surgery, and radical surgery were selected. The surgical method was used as the grouping basis, and age, gender, BMl, ASA, and TNM stages were used as covariables to calculate the propensity score. The patients with the closest scores in the two groups were paired for comparison. This made the comparability of the data more significant and illustrative.

Over the last 5 years, 671 rectal cancer surgeries were performed in our hospital, and 198 cases were over 70 years old. Among 198 cases, 63 cases underwent open surgery, and 135 cases underwent laparoscopic surgery. After the screening for exclusion and inclusion criteria and matching, 51 pairs of data were obtained. No difference in age, gender, BMI, ASA, and TNM staging were found between groups. Francesco et al ${ }^{[21]}$ compared rectal cancer patients over 80 years old with those between 60 and 69 years old and found that the average $\mathrm{CCl}$ of patients over 80 years old was 7 , and the average $\mathrm{CCl}$ of patients aged $60-69$ years old was $4(P=0.001)$. The ASA score in the older age group was also significantly higher. These data are consistent with our results. Only $1 / 4$ of patients in the two groups $(25.5 \%$ vs. $23.5 \%)$ had no combined disease $(\mathrm{CCl}=0)$. The two groups' ASA scores were also higher, which indicated that older patients had more comorbidities and had poor physical fitness. This was also the main reason for the high risk of surgery.

Previous studies had shown that laparoscopic rectal surgery has obvious minimally invasive advantages compared with open surgery. The patients who underwent laparoscopy showed less intraoperative bleeding, faster recovery of gastrointestinal function, and shorter hospital stay ${ }^{[22]}$. In this study, we observed a similar phenomenon in older patients. Patients in the LS group had less intraoperative blood loss, faster recovery of intestinal function, early first exhaust time, less need for postoperative analgesics, and shorter hospital stay. This suggested that in older patients with rectal cancer, laparoscopic surgery may lead to less stress, less pain, and faster recovery. However, LS required a longer operation time than OS (193 minutes vs. 203 minutes) $(P=0.048)$. Similar results were observed in previous studies. Akiyoshi et al ${ }^{[23]}$ observed consecutive cases of rectal cancer and divided them into three groups: laparoscopic surgery group with age $>75$ years old $(n=44)$, laparoscopic surgery group with age $<75$ years old $(n=$ $228)$, and the open surgery group with age $>75$ years old $(n=44)$. The results showed that laparoscopic surgery lasted significantly longer than open surgery (256 minutes vs. 196 minutes). This, in turn, indicated that laparoscopy is a highly demanding surgical technique that puts more time and energy 
strain on surgeons, while patients also need to undergo longer surgery and anesthesia. However, in our study, the average time difference between the two groups was only 20 minutes. We believe that this difference may be due to different surgeons, but it was more likely to indicate an improvement in technology. With the development of the technology and the improvement of the surgeon's proficiency, LS and OS's time gap will gradually decrease. Nonetheless, laparoscopic surgery may become a preferred surgery method due to speed and efficiency.

In our study, the LS group had a lower incidence of complications than the OS group. The total cases of postoperative complications of the two groups were 11 (21.6\%) and 18 (35.3\%), respectively, with no statistical difference $(P=0.124)$. However, the tendency of anastomotic complications in the LS group was higher than that in the OS group (7.8\% vs. $4 \%)$, and the tendency of incision-related complications in the OS group was higher than that in the LS group (11.7\% vs. $2 \%)$. Paralytic ileus was more common in OS group (7.8\% vs. $3.9 \%)$, while cardiopulmonary complications were observed in both groups ( $7.8 \%$ vs. $11.7 \%)$. However, no significant statistical difference between the two groups was found. In addition, no death occurred within 30 days of operation in both groups. The information obtained by this study was consistent with previous studies ${ }^{[16,18,23]}$. We observed the situation of older patients, and there were more cardiopulmonary complications after surgery, which may be related to the reduced cardiopulmonary reserve and the reduced ability to withstand surgical strikes in older patients. There were many incisionrelated complications in open surgery, which were associated with large incisions and longer operation time through the incision. A small incision in laparoscopic surgery was only used for specimen removal and short operation time, which may be the main reason for the fewer incision complications.

As for the anastomose-related complications, we combined TDAV and PO for further analysis. In general, the Dixon proportion in the LS group was similar to that in the OS group ( $30 \%$ vs. $27 \%, P=0.786)$, and the sphincter preserving rates of the LS group and OS group were basically the same. Nevertheless, in the TDAV subgroup study, we found that in the Dixon surgery with anal preservation, for low rectal cancer with TDAV $<5 \mathrm{~cm}$, more patients tended to choose LS ( $42.5 \%$ vs. $21.6 \%, P=0.051)$; for middle rectal cancer with TDAV of $5-10 \mathrm{~cm}$, more patients tended to choose OS ( $27.5 \%$ vs. $51.4 \%, P=0.032)$; for high rectal cancer with TDAV $>10 \mathrm{~cm}$, LS and OS were selected in a similar proportion (30\% vs. $27 \%, \mathrm{P}=$ 0.773). In low rectal cancer with $T D A V<5 \mathrm{~cm}$, the ratio of $P O$ of $L S$ was higher than that of $O S(52.9 \%$ vs. $37.5 \%, P=0.082)$, but there was no significant statistical difference. In the middle rectal cancer with TDAV of 5-10 cm, the PO ratio between the two groups was similar $(18.1 \%$ vs. $10.5 \%, P=0.936)$. In high rectal cancer with TDAV $>10 \mathrm{~cm}$, neither group had PO. From the above data, we can see that the tumor's location may be an important reason for the surgeon's choice. In patients with low tumor location, surgeons were more likely to choose LS. The magnifying effect and fine operation of the laparoscope in narrow space can help surgeons to complete the operation better. In open surgery, the tumor location is generally higher, and anastomosis after resection is easier to achieve, which may account for the low anastomosis-related complications observed during open surgery. Still, the low position operation also makes suturing after anastomosis very difficult. The uncertainty of low position surgery might drive 
surgeons to choose PO more so as to balance and make up for the shortcomings of surgery, thus reducing the risk of surgery. This may be the reason for the high proportion of PO in LS.

An average follow-up time was 35.7 months (7-59 months). Survival rates were compared using the Logrank test. Statistical analysis showed no statistical difference between the two groups $(X 2=0.198, P=$ $0.656)$. This indicated that there was no significant difference in the long-term effect in older patients between the two surgical methods.

This study has a few limitations. It is a retrospective, non-randomized study with relatively small sample size. Different surgeons performed the operation, which can cause some bias. For older patients with complex conditions, the choice of OS or LS and whether PO was needed was related to the operator's assessment of the patient's condition and personal judgment, and there was obvious uncertainty. In the future, prospective randomized studies comparing LS and OS in older patients with rectal cancer should be conducted to confirm these findings.

Our results suggested that laparoscopic rectal cancer surgery has significant short-term advantages over open surgery in older patients, as it leads to less trauma, less pain, faster recovery. Laparoscopic rectal cancer surgery was associated with a shorter postoperative hospital stay, lower incidence of postoperative complications and, as well as a longer operating time. Age did not result as a risk or limitation factor for LS. Yet, more prospective comparative studies with a larger sample size are needed to confirm these findings.

\section{Abbreviations}

LS

laparoscopic surgery

OS

open surgery

$\mathrm{BMI}$

body mass index

ASA

American Society of Anesthesiologists

TDAV

Tumor Distance from the Anal Verge

$\mathrm{CCl}$

Charlson Comorbidity Index

PO

preventative ostomy

EBL

estimated blood loss 


\section{Declarations}

\section{Acknowledgement}

The authors thank all the investigators and staff who contributed to the patient follow-up and data collection in Capital Medical University Affiliated Beijing Shijitan Hospital.

\section{Statement of data availability}

All data generated or analyzed during this study are included in this article.

\section{Funding statement}

This research was supported by grants from the Beijing Municipal Administration of Hospital's Clinical Medicine Development of Special Funding Support (No. XMLX201708). We give thanks here.

\section{Conflict of interest disclose}

Qiang Li, Hong gao, Jin-zhong Wang, Yu-bin Zhu have no conflicts of interest or financial ties to disclose.

\section{Ethics approval statement}

The study protocol has been approved by The Ethics Committee for Scientific Research of Capital Medical University Affiliated Beijing Shijitan Hospital.

\section{Patient consent statement}

In this reach, the subjects have given their informed consent.

\section{Author Contributions}

Jin-zhong Wang and Yu-bin Zhu collected and analysed the data. Qiang Li and Jin-zhong Wang performed statistical analysis. Qiang Li and Yu-bin Zhu drafted the manuscript. Qiang Li and Hong gao performed the procedure, conceived of and designed the study, and critically revised all the intellectual content of the manuscript. All authors read and approved the final manuscript.

\section{Research involving human and animal participants}

This article does not contain any studies with human participants or animals performed by any of the authors.

\section{References}

1. Jemal A, Bray F, Center MM, Ferlay J, Ward E. Forman D(2011)Global cancer statistics. CA Cancer J Clin 61:69-90. 
2. Levi F, Lucchini F, Negri E, Boyle P, La Vecchia C. Changed trends of cancer mortality in the elderly. Ann Oncol. 2001;12(10):1467-77.

3. Ferlay J, Soerjomataram I, Dikshit R, et al. Cancer incidence and mortality worldwide: sources, methods and major patterns in GLOBOCAN 2012. Int J Cancer. 2015;136(5):E359-86.

4. Athanasiou CD, Robinson J, Yiasemidou M, Lockwood S, Markides GA. Laparoscopic vs open approach for transverse colon cancer. A systematic review and meta-analysis of short and long term outcomes. Int J Surg. 2017;41:78-85.

5. Parker JM, Feldmann TF, Cologne KG. Advances in Laparoscopic Colorectal Surgery. Surg Clin North Am. 2017 Jun;97(3):547-60.

6. Frasson M, Braga M, Vignali A, Zuliani W, Di Carlo V. Benefits of laparoscopic colorectal resection are more pronounced in elderly patients. Dis Colon Rectum. 2008 Mar;51(3):296-300.

7. Kearney DE, Coffey JC. A Randomized Trial of Laparoscopic versus Open Surgery for Rectal Cancer. N Engl J Med. 2015 Jul;9(2):194. 373(.

8. Green BL, Marshall HC, Collinson F, Quirke P, Guillou P, Jayne DG, Brown JM. Long-term follow-up of the Medical Research Council CLASICC trial of conventional versus laparoscopically assisted resection in colorectal cancer. Br J Surg. 2013 Jan;100(1):75-82.

9. Zimmermann M, Merkel S, Weber K, Bruch HP, Hohenberger W, Keck T, Grützmann R. Laparoscopic surgery for rectal cancer reveals comparable oncological outcome even in context of worse shortterm results-long-term analysis of nearly 500 patients from two high-volume centers. Int $\mathrm{J}$ Colorectal Dis. 2019 Sep;34(9):1541-50.

10. Story DA. Postoperative complications in elderly patients and their significance for long-term prognosis. Curr Opin Anaesthesiol. 2008 Jun;21(3):375-9.

11. Shahidi S, Schroeder TV, Carstensen M, Sillesen H. Outcome and survival of patients aged 75 years and older compared to younger patients after ruptured abdominal aortic aneurysm repair: do the results justify the effort? Ann Vasc Surg. 2009 Jul-Aug;23(4):469-77.

12. Story DA. Postoperative mortality and complications. Best Pract Res Clin Anaesthesiol. 2011 Sep;25(3):319-27.

13. Charlson M, Szatrowski TP, Peterson J, Gold J. Validation of a combined comorbidity index. J Clin Epidemiol. 1994 Nov;47(11):1245-51.

14. Howlader N, Noone AM, Krapcho M, Garshell J, Miller D, Altekruse SF, Kosary CL, Yu M, Ruhl J, Tatalovich Z, Mariotto A, Lewis DR, Chen HS, Feuer EJ, Cronin KA. (2015) SEER Cancer Stat Rev 1975-2011. National Cancer Institute, Bethesda, MD. http://seer.cancer.gov/csr/1975_2011/. Updated December 17, 2014.

15. Devoto L, Celentano V, Cohen R, Khan J, Chand M. Colorectal cancer surgery in the very elderly patient: a systematic review of laparoscopic versus open colorectal resection. Int J Colorectal Dis. 2017 Sep;32(9):1237-42.

16. Arenal-Vera JJ, Tinoco-Carrasco C, del-Villar-Negro A, Labarga-Rodríguez F, Delgado-Mucientes A, Cítores MA. Colorectal cancer in the elderly: characteristics and short term results. Rev Esp Enferm 
Dig. 2011 Aug;103(8):408-15. English, Spanish.

17. Jayne DG, Thorpe HC, Copeland J, Quirke P, Brown JM, Guillou PJ. Five-year follow-up of the Medical Research Council CLASICC trial of laparoscopically assisted versus open surgery for colorectal cancer. Br J Surg. 2010 Nov;97(11):1638-45.

18. Hiki N, Shimizu N, Yamaguchi H, Imamura K, Kami K, Kubota K, Kaminishi M. Manipulation of the small intestine as a cause of the increased inflammatory response after open compared with laparoscopic surgery. Br J Surg. 2006 Feb;93(2):195-204.

19. Schiphorst AH, Pronk A, Borel Rinkes IH, Hamaker ME. Representation of the elderly in trials of laparoscopic surgery for colorectal cancer. Colorectal Dis. 2014 Dec;16(12):976-83.

20. Roscio F, Boni L, Clerici F, Frattini P, Cassinotti E, Scandroglio I. Is laparoscopic surgery really effective for the treatment of colon and rectal cancer in very elderly over 80 years old? A prospective multicentric case-control assessment. Surg Endosc. 2016 Oct;30(10):4372-82. doi:10.1007/s00464016-4755-7. Epub 2016 Feb 19. PMID: 26895891.

21. Vignali A, Di Palo S, Tamburini A, Radaelli G, Orsenigo E, Staudacher C. Laparoscopic vs. open colectomies in octogenarians: a case-matched control study. Dis Colon Rectum. 2005 Nov;48(11):2070-5.

22. Akiyoshi T, Kuroyanagi H, Oya M, Konishi T, Fukuda M, Fujimoto Y, Ueno M, Yamaguchi T. Short-term outcomes of laparoscopic rectal surgery for primary rectal cancer in elderly patients: is it safe and beneficial? J Gastrointest Surg. 2009 Sep;13(9):1614-8.

\section{Figures}




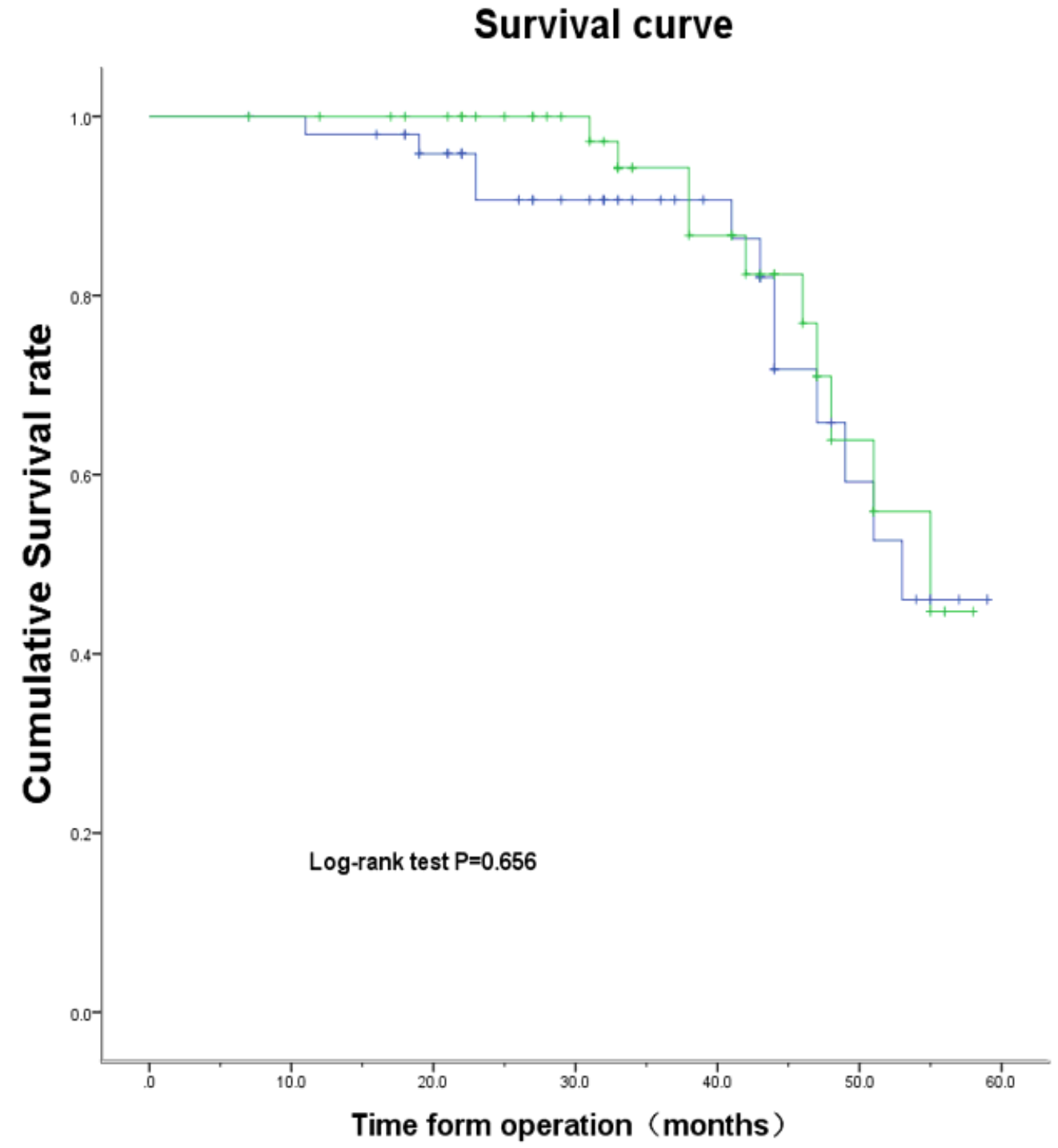

Operation type

$\neg$ Laparoscopic surgery

$\neg$ Open surgery

- Laparoscopic surgery-censored

+ Open surgery-censored

Time form operation (months)

Figure 1

Kaplan-Meier survival curves of the Laparoscopic Surgery group and the Open Surgery group. 